\title{
Displacive Phase Transformations and Generalized Stacking Faults
}

\author{
V. PAidar ${ }^{a, *}$, A. Ostapovets ${ }^{a}$, O. Hardouin Duparc $^{b}$ \\ AND O. KHALFALLAH ${ }^{c}$ \\ ${ }^{a}$ Institute of Physics AS CR, Prague, Czech Republic \\ ${ }^{b}$ LSI, Ecole Polytechnique, Palaiseau, France \\ ${ }^{c}$ LMDM, Mentouri University, Constantine, Algeria
}

\begin{abstract}
The displacive phase transformations can be considered as composed of two processes, namely, pure displacements, shuffling or shearing of atomic planes, and supplementary homogeneous lattice deformation changing also the dimensions of the moving planes. Such deformation causes shape memory effect when the structural transformation is reversed. General displacements of atomic planes will be examined, i.e. $\gamma$-surface type calculations will be reported for single plane shuffling, alternate shuffling of every other bcc atomic plane and successive displacements of parallel atomic planes producing in combination with homogeneous deformation the close packed structures. The results of calculations using the many-body potentials of the Finnis-Sinclair type will be compared with $a b$ initio calculations that indicate in which way the phase transformation can be initiated.
\end{abstract}

PACS: $63.70 .+\mathrm{h}$

\section{Introduction}

In martensitic transformations the high symmetry austenite phase is converted into a structure of lower symmetry by displacive mechanism. Several types of models on the macroscopic and mesoscopic levels were developed in the literature to describe the resulting structures and their properties [1]. Nevertheless, the underlying processes on the atomic level are not yet fully understood. Hence theoretical approaches taking into account atomic bonding in addition to the geometrical aspects of transformation processes are highly needed.

High temperature austenitic phases that are often bcc based $\left(\mathrm{B2}, \mathrm{DO}_{3}\right.$ or $\left.\mathrm{L2}_{1}\right)$ intermetallics transform into more close-packed martensitic structures that may have lattices similar to hcp or fcc. The fundamental aspects of transformation mechanisms for mono-elemental structures are discussed in this paper to elucidate what roles are played by different processes. Shape memory materials are often multi-component ordered alloys, however, this type of complexity will be disregarded in this initial stage of our simplified approach.

Shuffling of atomic planes and lattice shearing can be considered as elementary processes that are activated during the phase transformations to martensitic structures. Let us consider two basic planar defects [2]: generalized stacking fault (GSF) located in between neighbour-

\footnotetext{
* corresponding author; e-mail: paidar@fzu.cz
}

ing atomic planes and generalized plane shuffling (GPS) of a single atomic layer. Moreover, these planar defects can be taken as building bricks of three-dimensional homogeneous structures produced by lattice shear or alternate shuffling, respectively. Shear deformation arises as pile-up of GSF placed on every successive parallel atomic plane forming thus generalized shear deformation (GSD). Similarly, the shuffling of every second parallel atomic plane forms the three-dimensional structure of generalized alternate shuffling (GAS). These structures combined with essentially planar homogeneous deformation lead to the bcc-fcc or bcc-hcp transition, respectively [2]. Notice that an additional deformation of the same type is imperative in both cases. For all those defects the energies can be calculated as functions of general displacement on the selected atomic plane, so called $\gamma$-surfaces.

Hence, the process of phase transformation can be divided into two parts: (i) change of mutual positions of atomic planes, their stacking, preserving their internal atomic structure; (ii) transition of the bcc plane into the close-packed plane, (0001) in hcp or (111) in fcc with high density of atomic sites. The (i) process of cart mixing is purely displacive while (ii) represents the additional deformation.

The aim of this paper is to compare the results of semi-empirical calculations employing the many-body potential with $a b$ initio calculations based on quantum mechanics. In both cases the transitions between bcc, hcp and fcc structures are considered. 


\section{Models of interatomic forces}

The many-body potential of the Finnis-Sinclair type was fitted to the fcc crystal characterized by the cohesive energy of $2.95 \mathrm{eV}$ and the lattice parameter of $0.409 \mathrm{~nm}$, just to scale the energy and atomic spacing units, and the bonding attractive forces were varied to get bcc or hcp lattices more stable [3]. In this paper only potential q2 will be employed that gives the close-packed structures more stable. Moreover, the structural stability varies with the atomic volume and its value of $16.2 \times 10^{-3} \mathrm{~nm}^{3}$ was selected for the comparison with the $a b$ initio calculations. This volume lies in between the values for the equilibrium hcp structure and the transition state when the bcc and hcp structures possess the same energy.

The density functional theory (DFT) calculations were performed for zirconium using the Vienna Ab initio Simulation Package (VASP ${ }^{\mathrm{TM}}$ ) software [4-7]. The projector augmented wave (PAW) method [8] with the Perdew-Burke-Ernzerhof semilocal GGA hybrid functional (PBE) [9] was used. The employed pseudopotential is taken from the VASP library (PAW_PBE). The two $5 s$ electrons and the two $4 d$ ones are considered as valence ones and, thanks to the PAW method, the frozen core electrons are truly respected (they are not pseudized). The energies were computed with an energy cutoff of $250 \mathrm{eV}$ (versus VASP-PAW PBE's ENMAX which is $\approx 155 \mathrm{eV}$ ). The integration in the Brillouin zone was used considering the Monkhorst-Pack points $(11 \times 11 \times 11$ for the four atom boxes $)$ via the tetrahedron method with the Blöchl corrections [10].

\section{Cuts of $\gamma$-surfaces}

The cuts of GAS $\gamma$-surfaces for three-dimensional alternate shuffling along the [101] direction both without and with the additional deformation calculated using the q2 many-body potential (Fig. 1) and DFT-VASP code for $\mathrm{Zr}$ (Fig. 2) represent the trajectories for the bcc-hcp transitions. It is noteworthy that a new minimum appears in the $a b$ initio calculations already without the additional deformation (see Fig. 2) while for the many-body potential the minimum stays at the origin. That new minimum is not at the crystallographic point of hcp structure $(\boldsymbol{b} / 6)$, it is situated at about $\boldsymbol{b} / 10$. $\boldsymbol{b}$ is the lattice vector in the bcc [101] direction. The displacement of $\boldsymbol{b} / 2$ corresponds to the maxima of $\gamma$-surfaces without deformation [3]. The hcp minimum appears for the q2 potential only owing to the additional deformation, i.e. due to the rearrangements of atomic positions on the shuffling planes, the isosceles triangle of the first and second bcc neighbours on the $\{101\}$ plane is converted into the equilateral triangle of the close-packed structures on the $\{0001\}$ hcp or $\{111\}$ fcc planes. The displacive mechanism of phase transformations bcc-hcp is also discussed in [11].

Similarly also the cuts of GSD $\gamma$-surfaces for three-dimensional shearing along the [101] direction both without and with the additional deformation using the q2

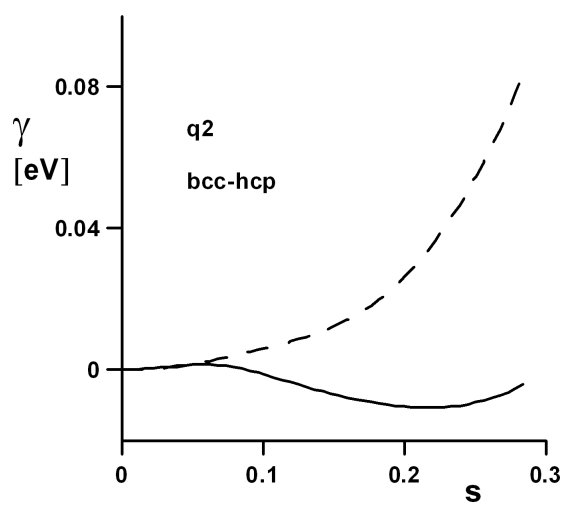

Fig. 1. Sections of GAS $\gamma$-surface for $q^{2}$ potential along the [101] direction with (full line) and without (dashed line) the additional deformation for the atomic volume of $16.2 \times 10^{-3} \mathrm{~nm}^{3}$. Displacements $s$ is $\sqrt{2}$ from the origin up to [101], $\boldsymbol{b}$.

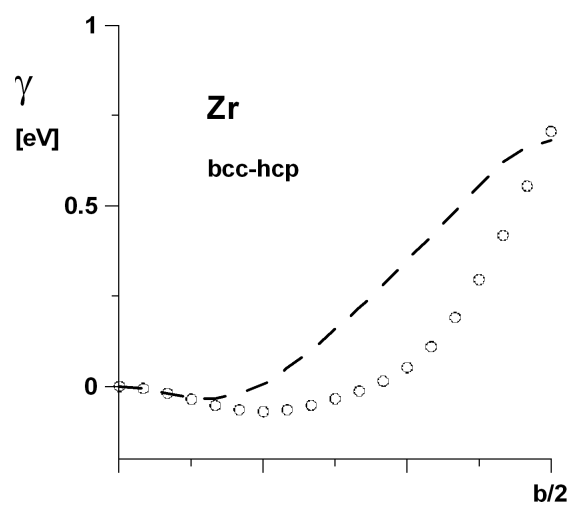

Fig. 2. Sections of GAS $\gamma$-surface for $\mathrm{Zr}$ ab initio calculations along the [101] direction with (circles) and without (dashed line) the additional deformation. Displacements from the origin up to about $1 / 2$ [101], $\boldsymbol{b} / 2$.

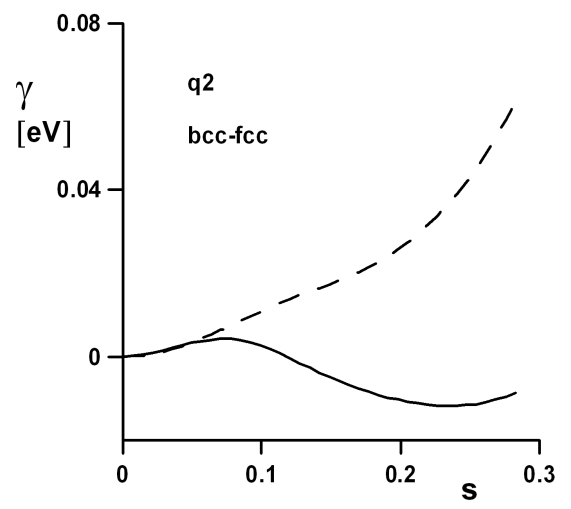

Fig. 3. Sections of GSD $\gamma$-surface for $q^{2}$ potential along the [101] direction with (full line) and without (dashed line) the additional deformation for the atomic volume of $16.2 \times 10^{-3} \mathrm{~nm}^{3}$. 


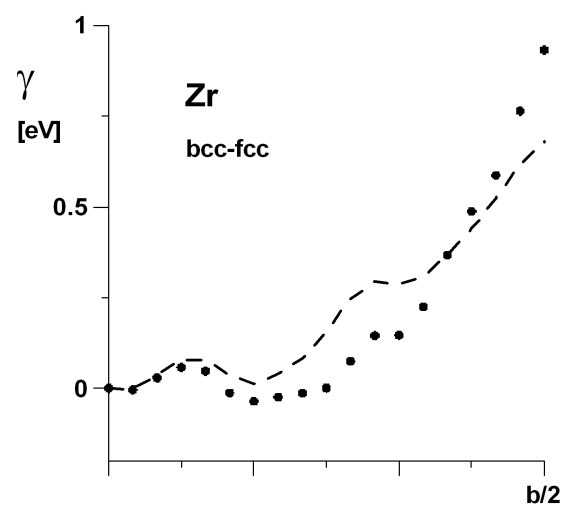

Fig. 4. Sections of GSD $\gamma$-surface for $\mathrm{Zr}$ ab initio calculations along the [101] direction with (circles) and without (dashed line) the additional deformation.

many-body potential (Fig. 3) and DFT-VASP code for $\mathrm{Zr}$ (Fig. 4) were calculated. Contrary to the transition path bcc-hcp shown in Figs. 1 and 2 there is a local maximum on the transition path bcc-fcc for both types of interatomic forces roughly at about $\boldsymbol{b} / 12$. Obviously, the low temperature phase in $\mathrm{Zr}$ is hexagonal with lower energy that the austenitic bcc phase and the transformation from the bcc to hcp structure does not need energy activation. The high temperature bcc phase is stabilized by the entropy term [12] that is not taken into account in this paper. On the other hand, the fcc phase can have a lower energy than bcc but is not so easily accessible.

\section{Conclusions}

In agreement with experiments the calculated hcp structure of $\mathrm{Zr}$ is a more stable phase compared with the bcc as well as fcc phases even in the case when the DFT model is optimized for the bcc structure. The occurrence of a local minimum without the additional deformation indicates that the transition bcc-hcp is initiated by the atomic plane shuffling and the martensitic deformation leading to the fully developed closed-packed structure is sequential.
It is not surprising that the calculations with the q2 many-body potential are only in a qualitative agreement with the quantum mechanical model. This potential is not fitted to any particular element. It has been used only to show how a flexible semiempirical model can be different from a more physical DFT description of interatomic forces that is, however, applicable only to relatively small scale atomic configurations. Nevertheless, the effect of the additional deformation in the martensitic transformations has been clearly demonstrated in both cases.

\section{Acknowledgments}

Financial support of Grant Agency of ASCR, contract number IAA100100920, and of Grant Agency of ANR, contract ANR-10-BLAN_915 Fluti, is gratefully acknowledged.

\section{References}

[1] R.C. Pond, S. Celotto, J.P. Hirth, Acta Mater. 51, 5385 (2003).

[2] V. Paidar, Mater. Sci. Eng. A 481-2, 243 (2008).

[3] V. Paidar, A. Ostapovets, Solid State Phenom. 150, 159 (2009).

[4] G. Kresse, J. Hafner, Phys. Rev. B 47, 558 (1993).

[5] G. Kresse, J. Hafner, Phys. Rev. B 49, 14251 (1994).

[6] G. Kresse, J. Furthmueller, Phys. Rev. B 54, 11169 (1996).

[7] G. Kresse, J. Furthmueller, Comput. Mater. Sci. 6, 15 (1996).

[8] P. Bloechl, Phys. Rev. B 50, 17953 (1994).

[9] J.P. Perdew, K. Burke, M. Ernzerhof, Phys. Rev. Lett. 77, 3865 (1996).

[10] P. Bloechl, O. Jepsen, O.K. Andersen, Phys. Rev. B 49, 16223 (1994).

[11] V. Paidar, Prog. Mater. Sci. 56, 841 (2011).

[12] J. Friedel, J. Phys. (France) 35, L-59 (1974). 